\title{
Briefing: Young Coastal Scientists and Engineers Conference 2011
}

\author{
Jennifer M. Brown ${ }^{1}$ Tim Chesher ${ }^{2}$ Andrew J. Beveridge ${ }^{3}$ \\ Siti H. Shafiai ${ }^{4}$ Rory B. O'Hara Murray ${ }^{5}$ \\ Thomas A.G. Smyth ${ }^{6}$ Lucy M. Bricheno ${ }^{7}$ Oliver Way $^{8}$ \\ ${ }^{1}$ Coastal Modeller, National Oceanography Centre, Liverpool, L3 5DA, UK. (Email: jebro@noc.ac.uk) \\ ${ }^{2}$ Manager, Coasts and Estuaries Group, HR Wallingford, Wallingford, Oxfordshire, OX10 8BA, UK. \\ ${ }^{3}$ Marine Scotland - Science, Scottish Government, Marine Laboratory, Aberdeen AB11 9DB (Email: andrew.beveridge@scotland.gsi.gov.uk) \\ ${ }_{5}^{4}$ PhD student, Department of Engineering, University of Liverpool, L69 3GQ, UK (Email: s.shafiai@liverpool.ac.uk) \\ ${ }^{5}$ PhD Student, School of Environmental Sciences, University of Liverpool, Liverpool, UK. (Currently a Marine Renewables Oceanographer in the \\ Oceanography Group, Marine Scotland-Science, Scottish Government, Marine Laboratory, Aberdeen AB11 9DB (Email: R.Murray@marlab.ac.uk) \\ ${ }^{6}$ PhD student, Centre for Coastal and Marine Research, University of Ulster University of Ulster, Coleraine, BT52 1SA (Email: smyth-ta@email.ulster.ac.uk) \\ ${ }^{7}$ Hydrodynamic-wave modeller, National Oceanography Centre, Liverpool, L3 5DA, UK (Email: luic@pol.ac.uk) \\ ${ }^{8}$ PhD Student, School of Ocean Sciences, Bangor University, Wales, LL59 5AH, UK (Email: ospa02@bangor.ac.uk
}

Proceedings of the ICE - Maritime Engineering, Volume 165, Issue 1, 01 March 2012.

\section{Abstract}

The $7^{\text {th }}$ UK Young Coastal Scientists and Engineers Conference welcomed 60 scientists and engineers to come together and discuss coastal research. The conference was hosted by the National Oceanography Centre in Liverpool, 30 - 31 March 2011. Early career practitioners and researchers presented 42 short papers on a range of topics driven by current issues addressed by UK universities, government agencies and consultancies. Although all presentations had a coastal theme, content varied from small scale processes to regional scale monitoring and management, covering topics such as structure impact, sediment transport, morphology, waves, currents, ecology, oxygen, estuarine systems, stratification, modelling, observation, ice, renewables and extreme events. This paper presents the keynote lecture and 6 abstracts considered to be of particular merit by the panel of judges. The conference continues to travel the UK and will be held at Bangor University in 2012.

\section{Keywords}

Maritime Engineering; Mathematical modelling; Field testing \& Monitoring 


\section{Briefings from the conference}




\section{Introduction - Jenny Brown}

The coast is a rapidly changing environment where the land meets the sea. Understanding coastal regions is of great importance and creates many challenges for scientists and engineers. Opportunities arise in navigation, fishing, minerals, energy, infrastructure, management and recreation. Complex coastal systems require improved understanding not only in the physical processes, such as hydrodynamics, waves, sediments and morphology, but also the social, economical, geological, geographical, biological and chemical processes. Both present and past conditions, along with future projections need to be understood to determine how best to let the coastline evolve under a changing climate and human development, and to defend assets against flooding and erosions. The impact studies for new development are also necessity to prevent unacceptable change or unsustainable cost to the environment. Multidisciplinary research is vital to improve understanding of the UK coastline and often involves modelling and observation, which requires development of knowledge, technologies and numerical methods. To promote an integrated UK coastal research community, the annual Young Coastal Scientists and Engineers Conference (YCSEC) started in 2005 at Nottingham and now travels the UK. This year the YCSEC went to Liverpool following previous events in Southampton, Plymouth, Oxford, Lancaster and London. In 2012 the conference will go to Bangor.

The YCSEC brings together early-career researchers (post-doctoral and research students) and practitioners in universities, government agencies and private companies. The YCSEC aims to provide a relatively informal setting for participants to experience conference conditions before attending more formal international research conferences. Participants present their work, maybe for the first time, in a friendly atmosphere in front of their peers and experienced scientists and engineers to gain valuable feedback. Delegates who have been in their post for 2 years or less are invited to present a poster, while delegates 3 years or more into their career are invited to give an oral presentation. New starters are also welcome to attend, in the hope they will return in following years to present. Participants are asked to submit an abstract in the form of a short paper. The presentations are judged by a panel made up from the YCSEC steering committee and prizes are awarded to the best oral and poster presentation. Since 2010, outstanding papers are selected for publication. This year 3 short papers from both presentation categories have been selected to form this proceeding. Sponsorship was sort to keep costs low to enable many UK students to attend. Delegates received a conference CD containing the book of abstracts. A conference dinner, in the heart of Liverpool's Cavern Quarter, and field trip, to Hilbre Island, were provided to enable ample opportunity to socialise and network in addition to breaks and over lunch. 


\section{Keynote Lecture: Coastal numerical modelling - plus ça change - Tim Chesher}

Over the last few decades, numerical modelling in the field of hydraulic civil engineering has come a long way, as evidenced from the changes that have taken place at HR Wallingford over its sixty year history.

When originally established, detailed hydraulic investigations were carried out at HR Wallingford using scale physical models, in combination with desk calculations where necessary. The only alternative at the time being full-scale field trials.

Gradually, and inexorably, however, physical modelling has given way to numerical modelling in many of the technical disciplines. There are various reasons for this development, including:

- Improvements in technical capabilities (chip speed, memory, and cost and availability of computers);

- A gradual, but real acceptance by both clients and third parties (e.g. auditors, regulators) of the benefits and capabilities of numerical modelling;

- Cost and convenience (e.g. archiving and re-commissioning). Whereas physical modelling requires space and specialist equipment (and once completed the models are typically removed to make way for the next study), numerical modelling has all the convenience of easy storage and retrieval;

- Knowledge gain (i.e. in theory) enabling equations to be written and solved with numerical code;

Development of analogues or alternative approaches to help where the theory is still lacking (e.g. Smooth Particle Hydraulics for two-phase flows).

At HR Wallingford physical modelling is still in demand and continues to play an important role, albeit for a smaller set of disciplines than in the past, including:

- structural design and testing;

- wave overtopping calculations;

vessel response and mooring problems;

n wave agitation (occasionally).

In time, all the above will eventually yield to the power of the microchip. That said, this does not necessary signal the complete redundancy for physical modelling, as evidenced from the fact that tidal flow physical modelling is currently being undertaken as this paper is written, for a specific project on the Thames Estuary - something that has not taken place for a number of years. But nevertheless, numerical modelling has become the accepted means of research and application in the field of hydraulic civil engineering, and has proved itself to be an excellent tool to aid in both engineering design such as in the optimisation of port layouts, and environmental impact. Examples of application include:

- Flow modelling (2D, 3D, density effects, wind effects, littoral currents);

- Wave modelling (propagation, agitation, etc);

- Transport modelling (thermal, sediment, WQ, oil, etc);

- Morphological modelling (integrating hydraulic and sediment transport);

- Scour, near-bed processes (fluidisation, etc);

- Ecological modelling.

In addition, advances in computational power have led to: 
- (Much) more detailed resolution, finer meshes, and incorporation of fine-detailed bathymetry (e.g. LiDAR);

- Adaptive meshes;

very accurate numerical schemes;

- Longer timescales for simulations (e.g. morphological evolution).

The papers presented at YCSEC (as well as at many other workshops and conferences) are testament to the advances and benefits of numerical modelling. Consequently, it's very tempting to conclude that numerical modelling is capable of providing solutions to all engineering problems. And whilst this may be true at the general (high-level) scale, the fact still remains that by design (all) models are simplifications of reality, they very often omit important processes, and they can misrepresent others, and they can be misused. Fundamentally, it should always be borne in mind that models remain a tool only, and should not be used as a means to bypass thinking and brainpower.

The growth in availability of numerical models has led to improved (though sometime incomplete) understanding by clients, regulators, and other third parties, but with this also lies the risk of misunderstanding. This can lead to a mindset of "well you have a model of this area, you must be able to predict what will happen if..." And ignorance or inexperience by the modeller when applying a model as a black box can reinforce this misunderstanding. Hence along with their accuracy and convenience, it can be extremely easy to generate misleading results with a numerical model. And the improvements in presentation and development of sophisticated graphics (e.g. GIS) can further lead to overconfidence in the predictions.

The author has personally seen misapplication of models (e.g. sediment transport) in environments outside their range of validity, such as the prediction of sediment transport under cyclones conditions (ie well outside the range of validity of the model's equations). And even though such misapplication may be acknowledged, it is very easy to begin to convince oneself that the predictions are accurate, particularly when further model runs by way of sensitivity testing only serves to strengthen the 'base case' simulation as the most realistic with the other results being presented as 'outliers'.

So even though computational modelling provides an excellent means of study, it is essential that the user:

n knows which processes are represented in the model (and those that are not);

- knows the range and limits of validity of application of the model;

- has a good understanding of the quality of the inputs;

- knows how accurate the model is, noting that there can be substantial numerical dispersion introduced as the differential equations get turned into something that the model can solve;

- views the results with healthy scepticism and should remain to be convinced that the model is accurately reproducing reality. Avoid (at all costs) the mindset of "well the model generated the answer so it must be right".

By way of example Figure 2.1 shows wave heights around a detached breakwater on a plane sloping beach, under normal wave attack. The pattern in the lee of the breakwater appears sensible, with wave energy spreading (diffracting) into the lee, and to many readers the model would appear to be relatively accurate. However, the model applied does not include diffraction effects, and other than that due to a little directional spreading, there should be no wave energy in the lee: it is predominantly numerically generated. Note further that if diffraction is then included in the model, there will be too much energy in the lee, and consequently obtaining the correct wave height pattern is a non-trivial exercise. This is just one of myriad 
examples of models giving apparently accurate results but not necessarily for the right reasons. In addition, the old maxim of rubbish in -rubbish out still holds true, and poor calibration ought to imply poor predictive capability.

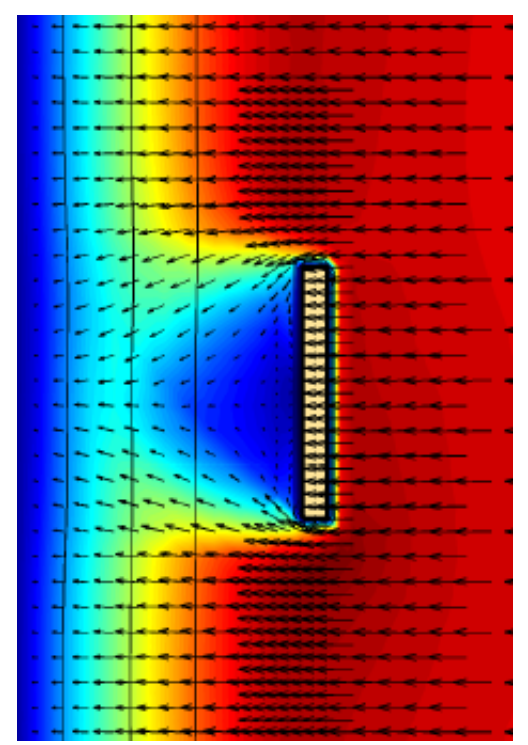

Figure 2.1 Wave heights around a detached breakwater on a plane sloping beach, under normal wave attack

So whilst it is clear that models are fantastically powerful tools that open up all sorts of possibilities in hydraulic civil engineering, as much as they develop and improve, the need to ensure diligence in application remains. Plus ça change. 


\section{Understanding the physical impact of demersal trawling activities - Andrew Beveridge}

\subsection{Introduction}

An understanding of the physical impact of towed fishing gears upon the seabed is of great importance in striving towards sustainable fisheries. Contact with the seabed is a necessary process in many demersal trawling operations; excessive contact, however, can damage benthic habitats, lead to benthic mortality, and increase the drag of the fishing gear, increasing the fuel costs to the trawler. Therefore developing an understanding of the process can suggest operational and design changes that can offer benefits to both the benthic habitat and to the fishing operation; increasing the sustainability.

\subsection{Methods}

A series of studies have been carried out at Marine Scotland Science and the University of Aberdeen in conjunction with SINTEF (Fisheries and Aquaculture). These include the development of numerical simulation techniques to predict the loads and penetrations of different fishing gear components. The numerical simulations are carried out using the engineering simulation package ABAQUS which uses the finite element method to calculate the loads and penetrations that occur during gear impact. Run in conjunction with the numerical simulations have been sea trails, where the drag force of full scale gear components have been ascertained to verify the numerical simulations (Ivanovic et al., In press). A lack of good agreement between sea trails and simulation predictions was found and therefore a new simulation technique was embarked upon. Utilising advances in the simulation package ABAQUS a Coupled EulerianLagrangian (CEL) approach was used. The CEL approach has previously been shown to be well suited to large deformation geomechanical problems (Qiu et al., 2011) and the impact of fishing gear components on the seabed is one such problem.

\subsection{Results and discussion}

The new simulation technique has given enhanced agreement between the sea trail data and simulation outputs with drag force predictions within $20 \%$ of the sea trail as opposed to $40 \%$ for the previous technique. Problems, however, still exist. It is difficult to match the true response of the seabed to the simulation. The simulation requires input data that has to be determined in a laboratory setting and in removing samples from the seabed the true response of the seabed, to loading, is lost. Future work to determine these values in situ would be advantageous to gaining an even greater accuracy from the simulations.

The final goal of having an accurate simulations technique is to have a generic tool that can be used to study the interaction of different fishing gear components, from a demersal trawl system, upon different seabed sediments. The simulations can be used to gain penetration and load data for the whole trawling system that can be related to ecological and environmental impact markers such as benthic mortality, nutrient release and sediment re-suspension along with trawler fuel consumption. Combined with spatial and temporal data for the whole demersal fishing fleet the ecological and environmental impact at a fleet level could be determined. Having this greater global understanding of demersal trawling activities will inform operational and design changes that can make the practice more sustainable. 


\subsection{References}

Ivanovic, A., Neilson, R.D. \& O'Neill, F.G. (In press). Modelling the physical impact of trawl components on the seabed and comparison with sea trials. Ocean Engineering, doi:10.1016/j.oceaneng.2010.09.011.

Qiu, G., Henke, S. \& Grabe, J. (2011). Application of a Coupled Eulerian-Lagrangian approach on geomechanical problems involving large deformations. Computers and Geotechnics, 38, No. 1, 3039. 


\section{The analysis of moving shoreline method in 2D lattice Boltzmann model for the long wave run-up in parabolic shaped basin - Siti Shafiai, Jian Zhou and Richard Burrows}

\subsection{Introduction}

Spontaneous geophysical activities such as underwater earthquakes and landslides could generate a massive and disastrous wave run-up. The impact could cause extensive flooding and loss of life. Many types of conventional numerical models have been developed and improved in order to simulate the wave run-up phenomenon (e.g. Briggs et al., 1995, Cho, 1995, Dodd, 1998, Fuhrman and Madsen, 2008, Kowalik and Bang, 1987, Lynett et al., 2002, Mahdavi and Talebbeydokhti, 2009, Synolakis, 1987, Titov and Synolakis, 1995, Yamazaki et al., 2009, Zelt, 1991). Although there are many approaches in the conventional numerical method available for wave run-up, very limited attempts have been made with the lattice Boltzmann method (LBM, Frandsen, 2008). Thus, a simple wave run-up model by using the LBM is developed.

\subsection{Mehods}

The space-filtered N-S equations with the large eddy simulation (LES) and subgrid-scale stress (SGS) model are used to elaborate the turbulent features in the current wave run-up lattice Boltzmann model. Two types of moving shoreline techniques: a) The thin film (Dodd, 1998, Oey, 2005) and b) The linear extrapolation (Lynett et al., 2002) are incorporated in the model. For validation, the results are compared with the experiments done by Thacker (1981).

\subsection{Results}

The water profile in the middle of the basin (in the $y$ direction) and along the $x$ direction at $t=0 T$ until $4 T+$ $1 / 2 T$ for the thin film model are plotted and compared in Figures 4.1-4.5. The numerical results showed a very good agreement to the analytic solutions. However, at $4 T$ and beyond, the model began to deviate (see Figure 5 (a) and (b)). For the linear extrapolation model, good results are achieved up to $T+1 / 2 T$. However, there were discrepancies from $2 T$ to beyond, where the simulated shapes of the wave are slightly under predicted. It is believed that this happened due to the lack of the dissipative term in the shallow water equation. The effect of the water profile deviation that is due to the dissipative term is strongly agreed with the previous analyses (Cho, 1995, Fuhrman and Madsen, 2008, Lynett et al., 2005).

\subsection{Discussion and conclusions}

The lattice Boltzmann model with moving shoreline for wave run-up was developed. Two types of moving shoreline were applied. The model capability in capturing the wave run-up was analyzed and validated by using appropriate analytical results. Overall, the thin film and linear extrapolation models have predicted the long-wave run-up phenomenon in a parabolic shaped basin excellently up to $4 T$ and $2 T$ cycle of period, respectively. The implementation of the thin layer of fluid in the whole computational domain resulted in a 
stable and simple model. The ability of the technique in preserving the mass and the momentum of the fluid during the computation are also proved in the previous analysis. Therefore, the thin film technique can be said to be the easiest and simple, yet accurate method that can be implemented in the LBM. Despite the results that are not satisfactory for the linear extrapolation model, overall, the thin film and extrapolation techniques are successfully applied in the current lattice Boltzmann model for capturing the moving shorelines.
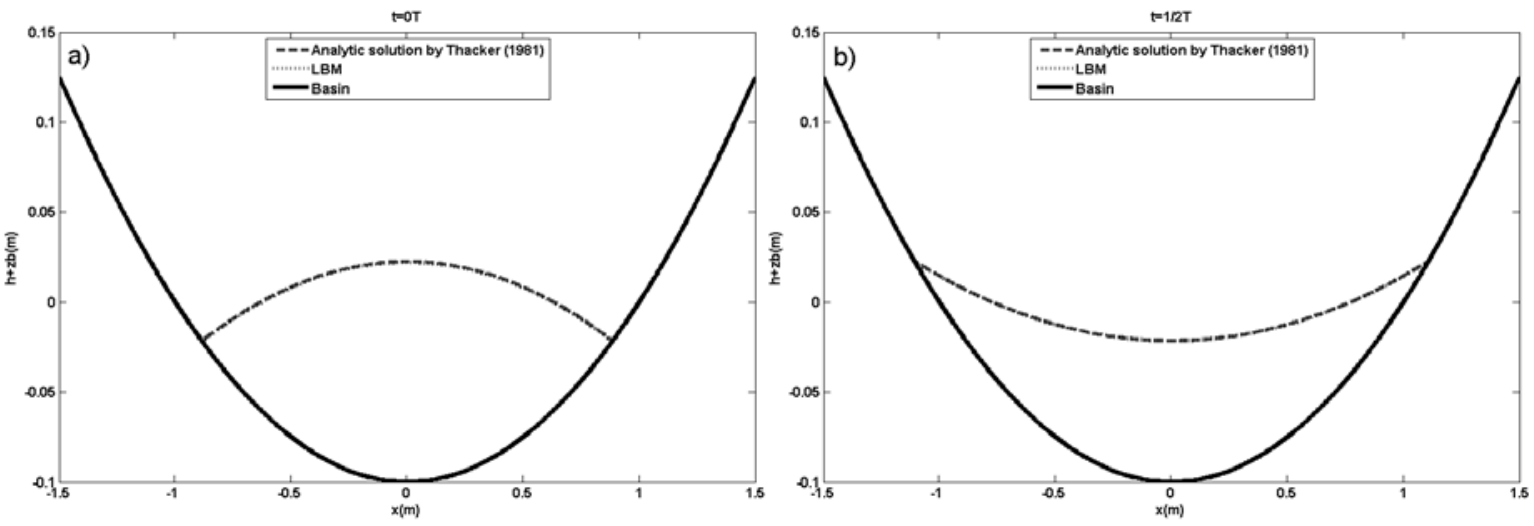

Figure 4.1 Parabolic free surface profiles oscillation for (a) $t=0 T$, (b) $t=0 T+1 / 2 T$.
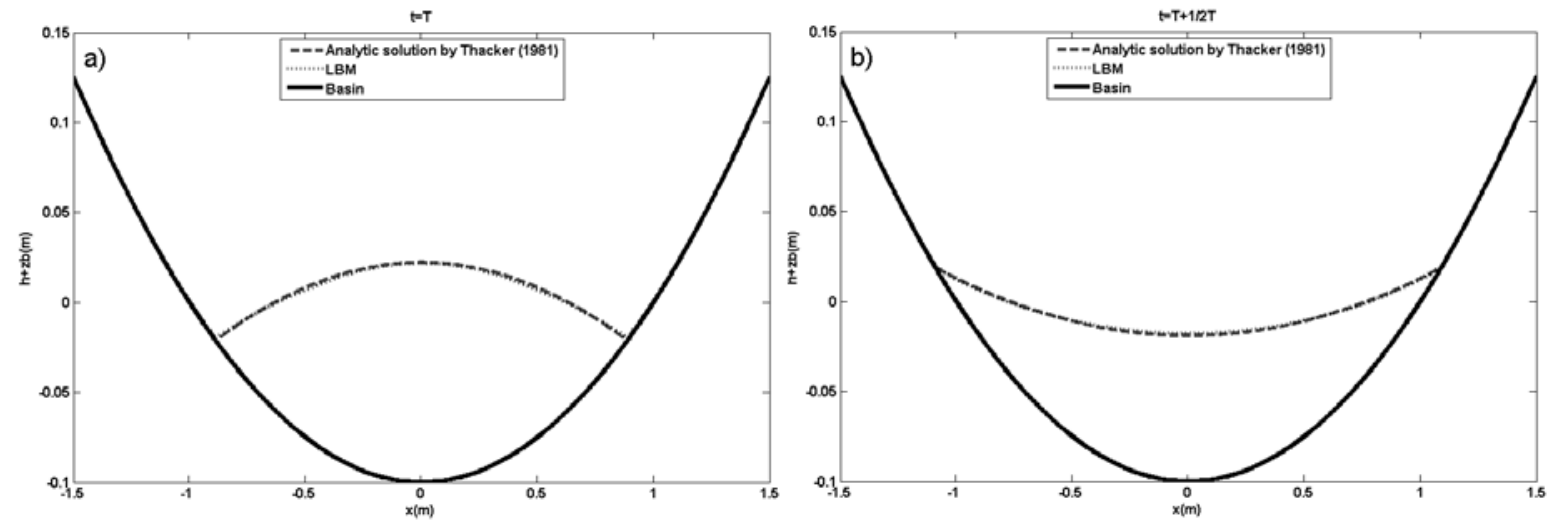

Figure 4.2 Parabolic free surface profiles oscillation for (a) $t=T$, (b) $t=T+1 / 2 T$.
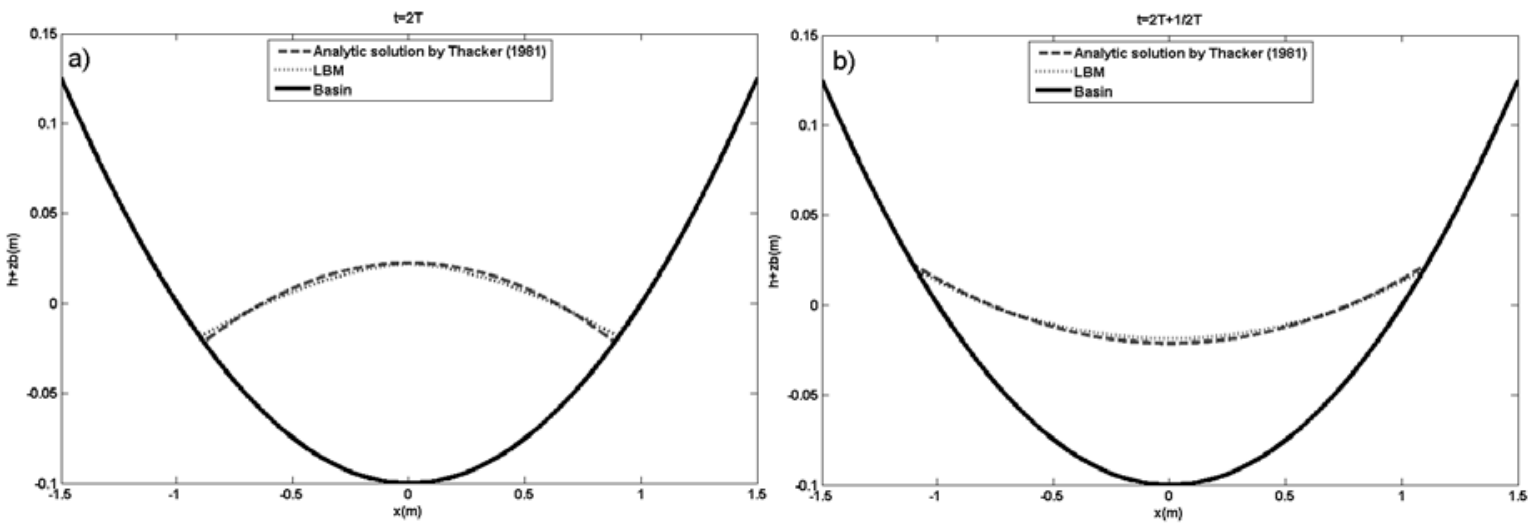

Figure 4.3 Parabolic free surface profiles oscillation for (a) $t=2 T$, (b) $t=2 T+1 / 2 T$ 

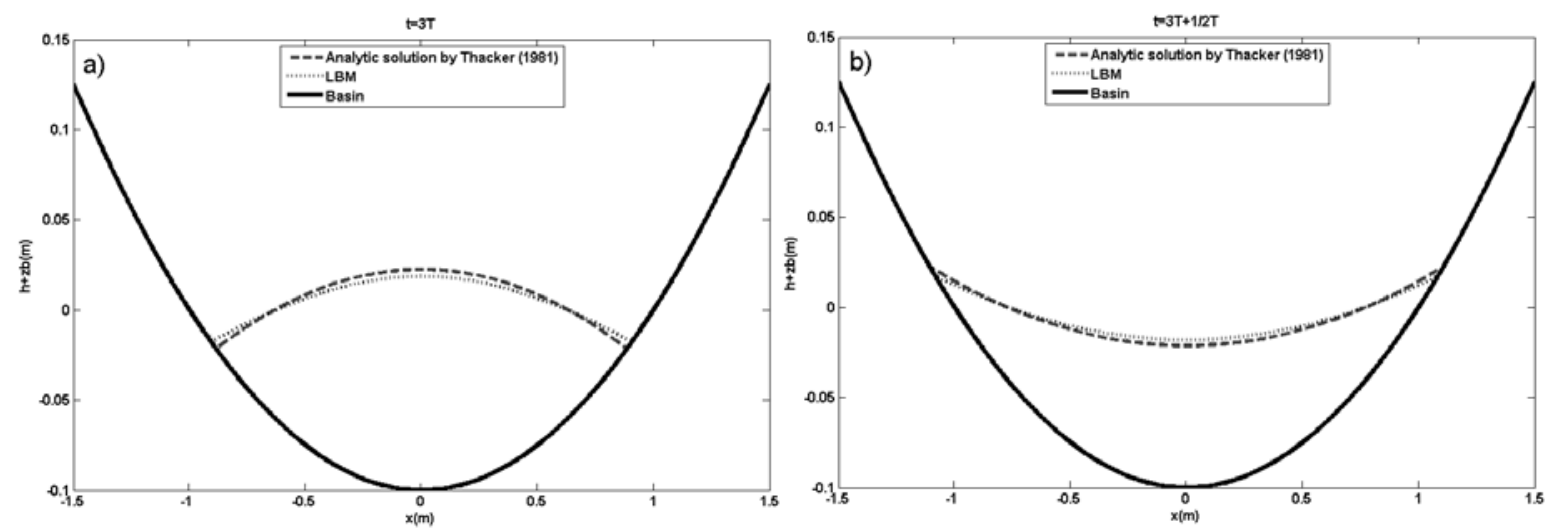

Figure 4.4 Parabolic free surface profiles oscillation for (a) $t=3 T$, (b) $t=3 T+1 / 2 T$.
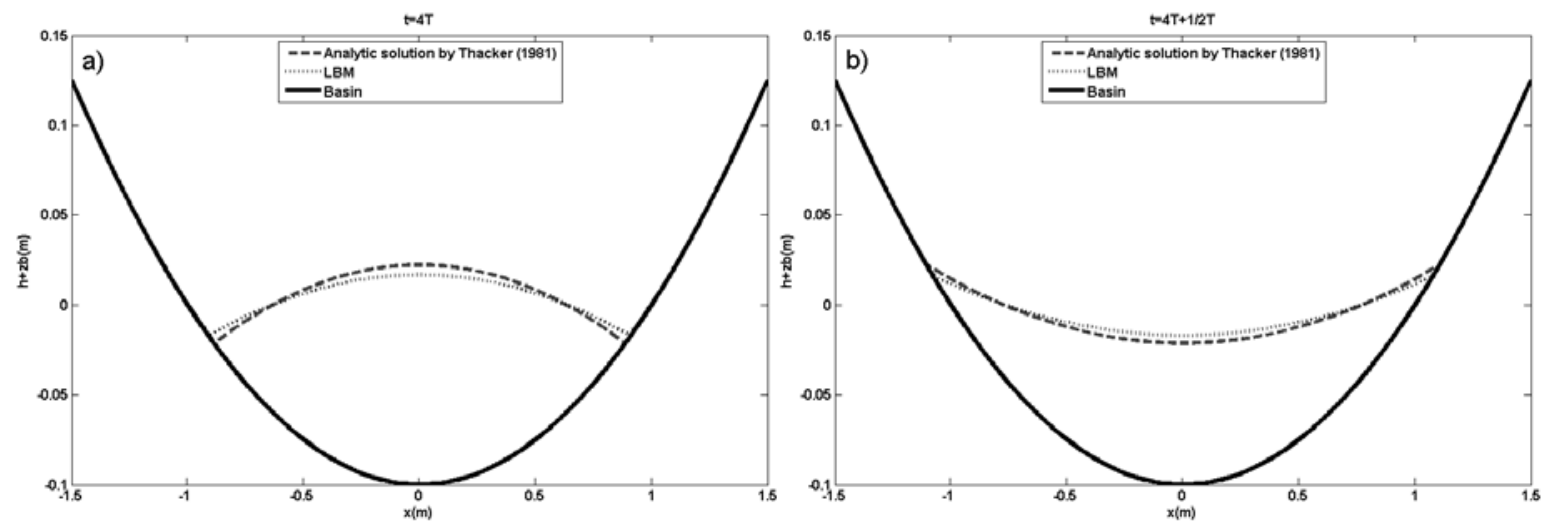

Figure 4.5 Parabolic free surface profiles oscillation for (a) $t=4 T$, (b) $t=4 T+1 / 2 T$.

\subsection{References}

Briggs, M.J., Synolakis, C.E., Harkins, G.S. \& Green, D.R. (1995). Laboratory experiments of tsunami runup on a circular island. Pure and Applied Geophysics, Earth and Environmental Science, 144, No. 3, 569-593.

Cho, Y.S. (1995). Numerical simulations of tsunami propagation and run-up. Cornell University, School of Civil and Environmental Engineering, NY, USA, Ph.D. Thesis. 256pp.

Dodd, N. (1998) Numerical model of wave run-up, overtopping, and regeneration. Journal of Waterway, Port, Coastal and Ocean Engineering, 124, No. 2, 73-81.

Fuhrman, D.R. \& Madsen, P.A. (2008). Simulation of nonlinear wave run-up with a high-order Boussinesq model. Coastal Engineering, 55, No. 2, 139-154.

Frandsen, J.B.A. (2008). Simple LBE wave runup model. Progress in Computational Fluid Dynamic, 8, No. $1-4,222-232$.

Kowalik, Z. \& Bang, I. (1987). Numerical computation of tsunami run-up by the upstream derivative method. Sci. Tsunami Hazards, 5, No. 2, 77-84.

Lynett, P.J., Wu, T. \& Liu, P.L.-F. (2002). Modeling wave runup with depth-integrated equations. Coastal Engineering, 46, No. 2, 89-107. 
Mahdavi, A. \& Talebbeydokhti, N. (2009). Modeling of non-breaking and breaking solitary wave run-up using FORCE-MUSCL scheme. Journal of Hydraulic Research, 47, No. 4, 476-485.

Oey, L.-Y. (2005). A wetting and drying scheme for POM. Ocean Modelling, 9, No. 2, 133-150.

Synolakis, C.E. (1987). The runup of solitary waves. Journal of Fluid Mechanics, 185:523-545.

Thacker, W.C. (1981). Some exact solutions to the nonlinear shallow-water wave equations. Journal of Fluid Mechanics, 107:499-508.

Titov, V.V. \& Synolakis, C.E. (1995). Modeling of breaking and nonbreaking long-wave evolution and runup using VTCS-2. Journal of Waterway, Port, Coastal \& Ocean Engineering - ASCE, 121, No. 6, 308 316.

Yamazaki, Y., Kowalik, Z. \& Cheung, K.F. (2009). Depth-integrated, non-hydrostatic model for wave breaking and run-up. International Journal for Numerical Methods in Fluids, 61, No. 5, 473-497.

Zelt, J.A. (1991). The run-up of nonbreaking and breaking solitary waves. Coastal Engineering, 15, No. 3, 205-246. 


\section{A stormy inheritance - bedform hysteresis and suspended sediment concentration - Rory O'Hara Murray, David Hodgson and Peter Thorne}

\subsection{Introduction}

The hydrodynamic forcing, the seabed, and the suspension of sediment together form a dynamic and interacting sediment transport triad (Thorne and Hanes, 2002). Therefore in a changing environment, typical of most near-shore zones, it is important to consider the interaction of all three components. Measurements are presented of the sediment transport triad of wave forcing, bedforms and suspended sediments during an experimental storm sequence, above a sandy bed, in a large scale flume facility. Under steep storm waves sheet flow conditions dominate and bedforms can be washed out (Davies and Thorne, 2008). As the waves reduce in steepness bedforms can start to reform, although relict washed out bedforms can remain (Hanes et al., 2001). The aim of this work is to examine whether such bedform hysteresis influences other parts of the sediment transport triad, such as the suspended sediment concentration (SSC).

\subsection{Methods}

The Deltaflume of Deltares Delft Hydraulics, the Netherlands, is a large scale flume $(230 \mathrm{~m}$ long, $5 \mathrm{~m}$ wide and $7 \mathrm{~m}$ deep) that enables sediment transport processes to be monitored at field scale. Irregular waves, with a JONSWAP spectrum (Carter, 1982), were generated above a sandy bed. A series of experiments were conducted during which the significant wave height, $H_{s}$, was systematically stepped up and down in order to simulate the waxing and waning of a storm sequence. Acoustic measurements were made of (i) the water velocities using an Acoustic Doppler Velocimeter (ADV); (ii) the bedforms cross-section using an Acoustic Ripple Profiler (ARP); (iii) the bedforms plan-form using a Sector Scanning Sonar (SSS); and (iv) time-average SSC profiles using an Acoustic Backscatter System (ABS).

\subsection{Results}

During the waxing phase of the storm sequence, where $H_{s}$ was stepped up systematically, twodimensional (2D) ripples, with long crest lines aligned perpendicular to the flow, emerged first and their steepness increased with $H_{s}$. At peak wave forcing three-dimensional (3D) ripples and hummocks formed. Figure 5.1 shows cross-sectional profiles of the bed obtained from the ARP under the waxing phase of the storm sequence. Plan-form geometry classifications of 2D, quasi-2D (q-2D), 3D and hummocks are indicated in Figure 5.1. When 2D ripples were present, the magnitude of the time-average SSC profiles broadly increased with $H_{s}$. In contrast, the SSC magnitude remained relatively constant above the 3D ripples and hummocks even though $H_{s}$ varied. During the waning phase of the storm sequence, where $H_{s}$ was stepped down systematically, the order of bedform emergence was broadly reversed with q-2D and $2 \mathrm{D}$ ripples reforming. There were, however, a number of characteristic differences between these beds and those that formed during the waxing phase of the storm sequence: (i) the spatial distribution of wavelengths and heights were somewhat larger; (ii) there were bedform development time lags; and (iii) there was some evidence of ripples being superimposed on longer wavelength bedforms. 


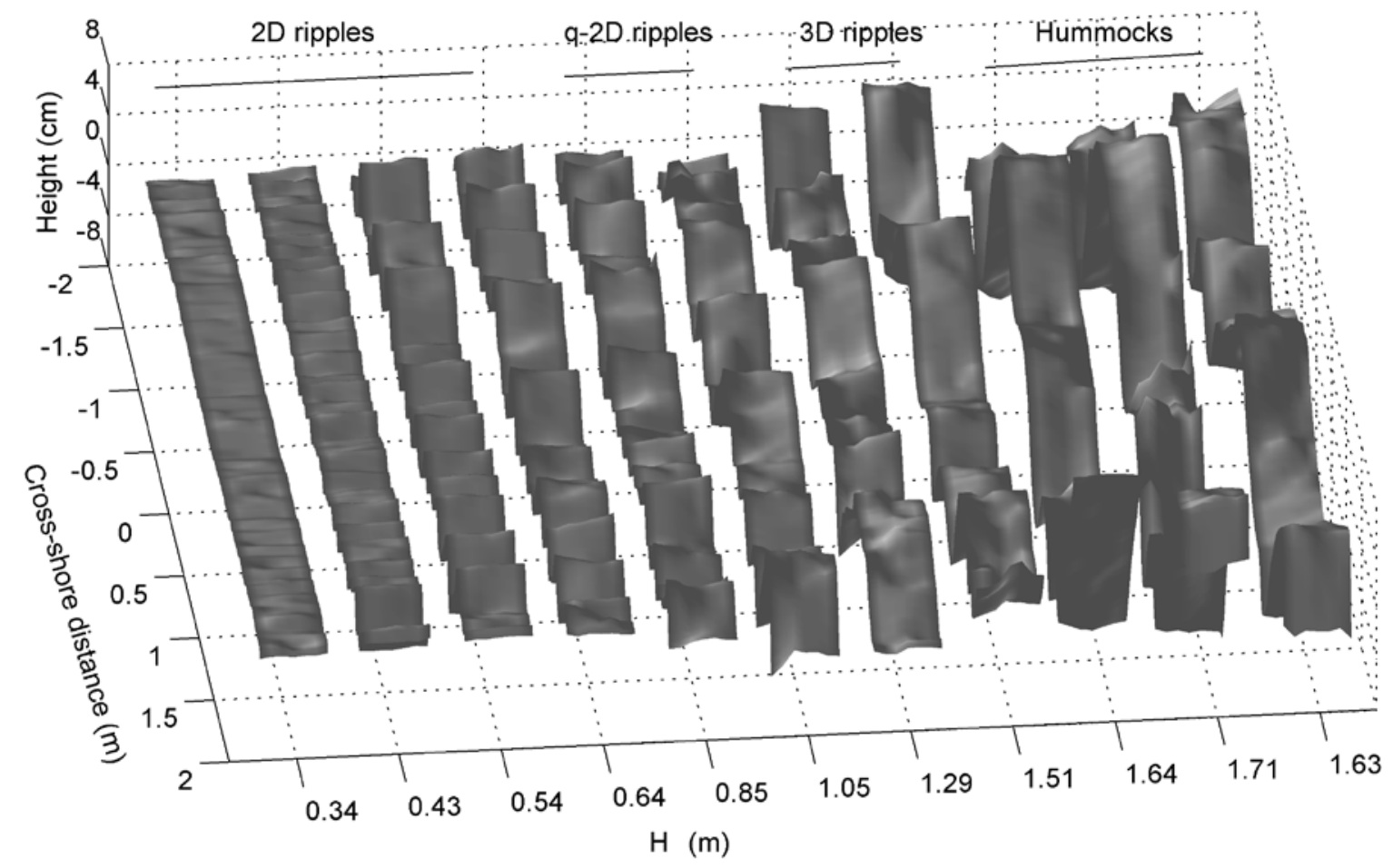

Figure 5.1 Results from the Acoustic Ripple Profiler depicting the response of the sandy bed to step changes in the significant wave height $\left(H_{s}\right)$.

\subsection{Discussion and conclusions}

Bedform hysteresis was observed during the waning phase of the storm sequence. Bedforms, being a component of a triad of sediments, can influence the time-average SSC. The prediction of time-average SSC is important for the calculation of net sediment fluxes in regional coastal settings. This work raises the possibility of there being differences in the SSC before and after a storm, despite the wave forcing conditions being the same.

\subsection{References}

Carter, D.J.T. (1982). Prediction of wave height and period for a constant wind velocity using the JONSWAP results. Ocean Engineering 9, No. 1, 17-33.

Davies, A.G. \& Thorne, P.D. (2008). Advances in the study of moving sediments and evolving seabeds. Surveys in Geophysics 29, No. 1, 1-36.

Hanes, D.M., Alymov, V., Chang, Y.S. \& Jette, C. (2001). Wave-formed sand ripples at Duck North Carolina. Journal of Geophysical Research - Oceans 106, No. C10, 22575-22592.

Thorne, P.D. \& Hanes, D.M. (2002). A review of acoustic measurement of small-scale sediment processes. Continental Shelf Research 22, No. 4, 60-632. 


\section{Comparison between modelled and recorded Three-Dimensional airflow over a coastal dune - Thomas Smyth, Derek Jackson and Andrew Cooper}

\subsection{Introduction}

Dune blowout enlargement is driven primarily by aeolian transport. High velocity winds entrain and remove sediment from the deflation basin, deepening the landform whilst simultaneously steepening the erosional walls, resulting in avalanching causing the blowout to grow laterally (Carter et al., 1990). Patterns of deflation in blowouts are however poorly understood, as near surface wind flow in a blowout is very complex. Previous research has shown that flow is topographically manipulated as it moves through the landform causing steering, reversal and jetting of the airflow (Hesp and Hyde, 1996, Hesp, 2002, Hansen et al. 2009, Hugenholtz and Wolfe, 2009). However, empirical data is scarce due to limited deployment of relatively sparse arrays of two dimensional anemometers and numerical approaches to flow behaviour appear to inexistent in the literature. In this study strong onshore winds (up to $24 \mathrm{~m} / \mathrm{s}$ ) were recorded using 24 ultrasonic anemometers in a north easterly orientated saucer blowout approximately $13 \mathrm{~m}$ deep, $60 \mathrm{~m}$ wide, and $100 \mathrm{~m}$ long from the throat to the depositional lobe. The blowout and surrounding area situated in the Belmullet Peninsula, Western Ireland, was also mapped and a $2 \mathrm{~m}$ resolution meshed surface generated over which a Computational Fluid Dynamics (CFD) model was performed.

\subsection{Methods}

Flow simulations were conducted over the dune using the average wind velocity and direction at the dune crest from a 40 minute period during the storm event. The two-equation Renormalised Group (RNG) kepilson model was run using the open source CFD software OpenFOAM. The mesh of the dune surface and surrounding area was generated using LiDAR data ( $6 \mathrm{~m}$ spot resolution) along with high resolution differential geographical positioning system (DGPS) surveys of less than $2 \mathrm{~m}$. During the survey, the heterogeneous aerodynamic roughness of the vegetation at the site was taken into consideration, and applied to the simulation by delineating areas of vegetation with a different roughness height from that of the bare sand and open water. Airflow data was collected using twenty four ultrasonic anemometers (3D Gill HS-50 model) at $50 \mathrm{~Hz} 1 \mathrm{~m}$ above the surface. The location of the anemometers was previously planned using the output from a simple CFD simulation of the site, identifying regions of wind flow steering and recirculation.

\subsection{Results}

The CFD results at $1 \mathrm{~m}$ above the surface (Figure 6.1) show that flow separates and reverses as it enters the blowout over the foredune crest. As it reattaches it is steered, diverging from the centre of the blowout, before topographically accelerating over the rim crest. Flow which enters the throat of the blowout accelerates and remains directionally unchanged before accelerating up the deposition lobe slope, where flow at the crest was simulated as being $100 \%$ faster at the crest than velocity simulated on the beach. Preliminary results from the anemometry data indicate that during the storm event airflow followed a very 
similar pattern to the simulated flow, with flow separation and reversal all occurring at the same points within the blowout. However the measured recirculation zones in lee of the dune crest and small ridge on the deflation basin appear to be much greater than those predicted, thus also increasing the distance before flow becomes reattached.

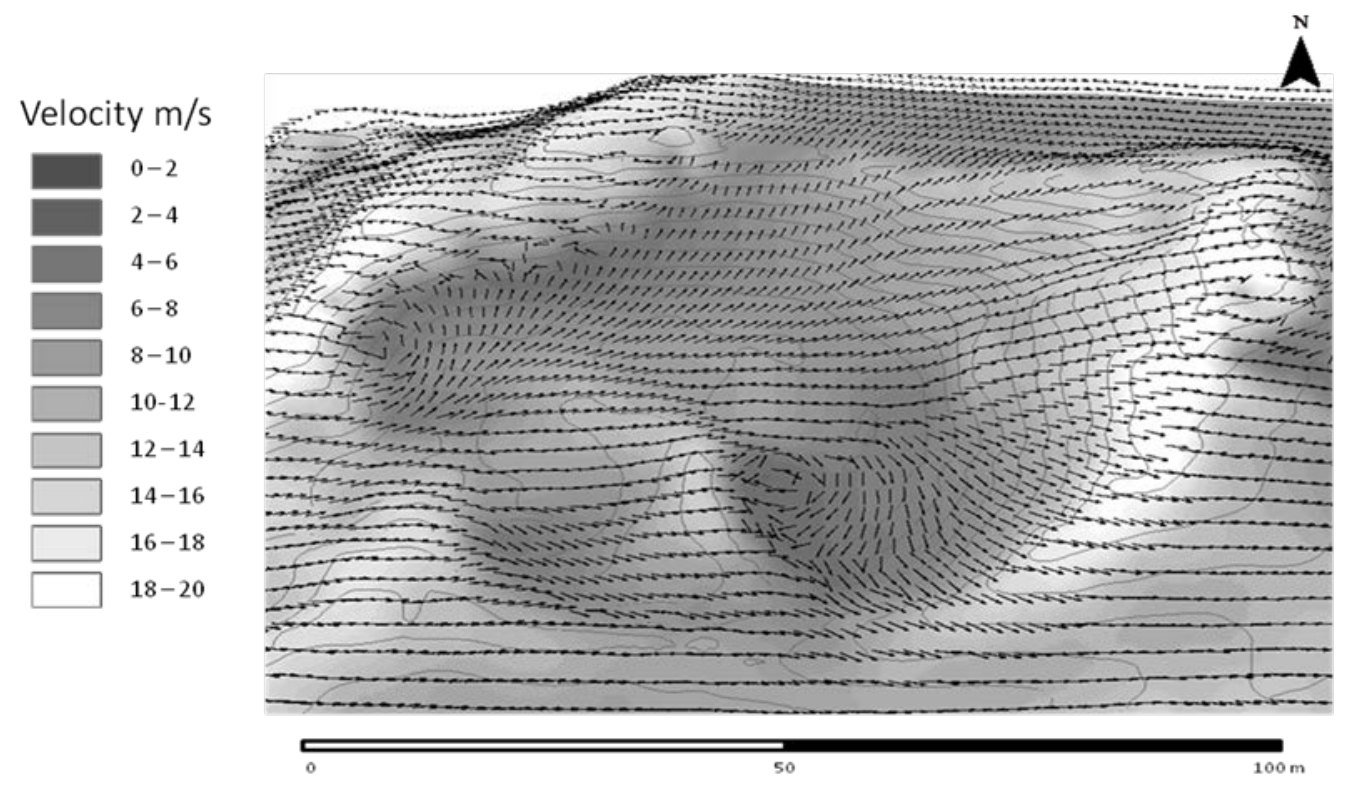

Figure 6.1 Airflow velocity vectors and velocity contours at $1 \mathrm{~m}$ over the topographic surface of the dune blowout

\subsection{Discussion and conclusions}

The comparison between simulated and measured data quantifies that regions of converging and diverging flow do occur within the blowout. As a consequence expansion could occur along all four walls of the blowout during a unidirectional storm event as flow becomes accelerated, separated and steered (Figure 6.1). Initial comparison between anemometry data and simulated flow does suggest that zones of flow reversal may be underestimated when simulated, however as both data sets are only in there preliminary stages of analysis more comparative studies are required make a more robust evaluation.

\subsection{References}

Carter, R.W.G, Hesp, P.A. \& Nordstrom, K.F. (1990). Erosional landforms in coastal dunes. In: Coastal Dunes: Form and Process (Nordstrom, K.F., Putsy, N.P. \& Carter R.W.G. (eds.)). Wiley, London, pp. 217-249.

Hesp, P.A. (2002). Foredunes and blowouts: Initiation, geomorphology and dynamics, Geomorphology, 48, No. 1-3, 245-268.

Hesp, P.A. \& Hyde, R. (1996). Flow dynamics and geomorphology of a trough blowout, Sedimentology, 43, No. 3, 505-525.

Hansen, E., Devries-Zimmerman, S., van Dijk, D. \& Yurk, B. (2009). Patterns of wind flow and aeolian deposition on a parabolic dune on the south eastern shore of Lake Michigan, Geomorphology, 105, No. 1-2, 147-157.

Hungenholtz, C.H., Wolfe, S.A. (2009). Form flow interactions in an aeolian saucer blowout, Earth Surface Processes and Landforms, 34, No. 7, 919-928. 


\section{The interaction of hydrodynamics, waves, and sediment transport in a coupled modelling system - Lucy Bricheno and Judith Wolf}

\subsection{Introduction}

The Proudman Oceanographic Laboratory Coastal Ocean Modelling System (POLCOMS) is a 3-D baroclinic hydrodynamic coastal ocean model. It is a state-of-the art tool for modelling tidal elevations and currents (Holt and James, 2001). A two-way coupling has been written, to combine POLCOMS with the spectral wave model WAM, leading to interesting advancements in our understanding of wave-current interaction. Several additional capabilities to POLCOMS have been included to give the model more scope. The physical model has been coupled with the European Seas Regional Ecosystem model (ERSEM), and it has also been used for sediment transport, fish larvae modelling and contaminant dispersion applications. In this work, we plan to strengthen the coupling between the wave and sediment transport modules, by considering the effects of waves on bottom shear stress. The sub-model for the simulation of suspended particulate matter (SPM) used is defined in Holt and James (1999).

\subsection{Methods}

In the original model, only the current stress was used to drive the sediment transport. However, as waves are known to be important intermittently, the wave stress is also calculated. The current stress is defined as $T_{c}=\rho C_{d} U_{b}{ }^{2}$ and the wave stress as $T_{w}=1 / 2 f_{w} U_{w}{ }^{2}$, where $\rho$ is the density of seawater, where $C_{d}$ and $f_{w}$ are the current and wave drag coefficients respectively, $U_{b}$ is the bottom (mean) current velocity and $U_{w}$ is the wave bottom orbital velocity amplitude The maximum combined stress $I_{\max }$ is defined in Soulsby (1997) as $I_{\max }=[($ $\left.\left.T_{c}+T_{w} \cos \varphi\right)^{2}+\left(T_{w} \sin \varphi\right)^{2}\right]^{1 / 2}$ where $\varphi$ is the difference between the wave and current directions. Both instantaneous and mean bed stresses must be taken into consideration, as the maximum (wave plus current) bed shear stress resuspends the bed sediment, whereas the mean stress transports SPM.
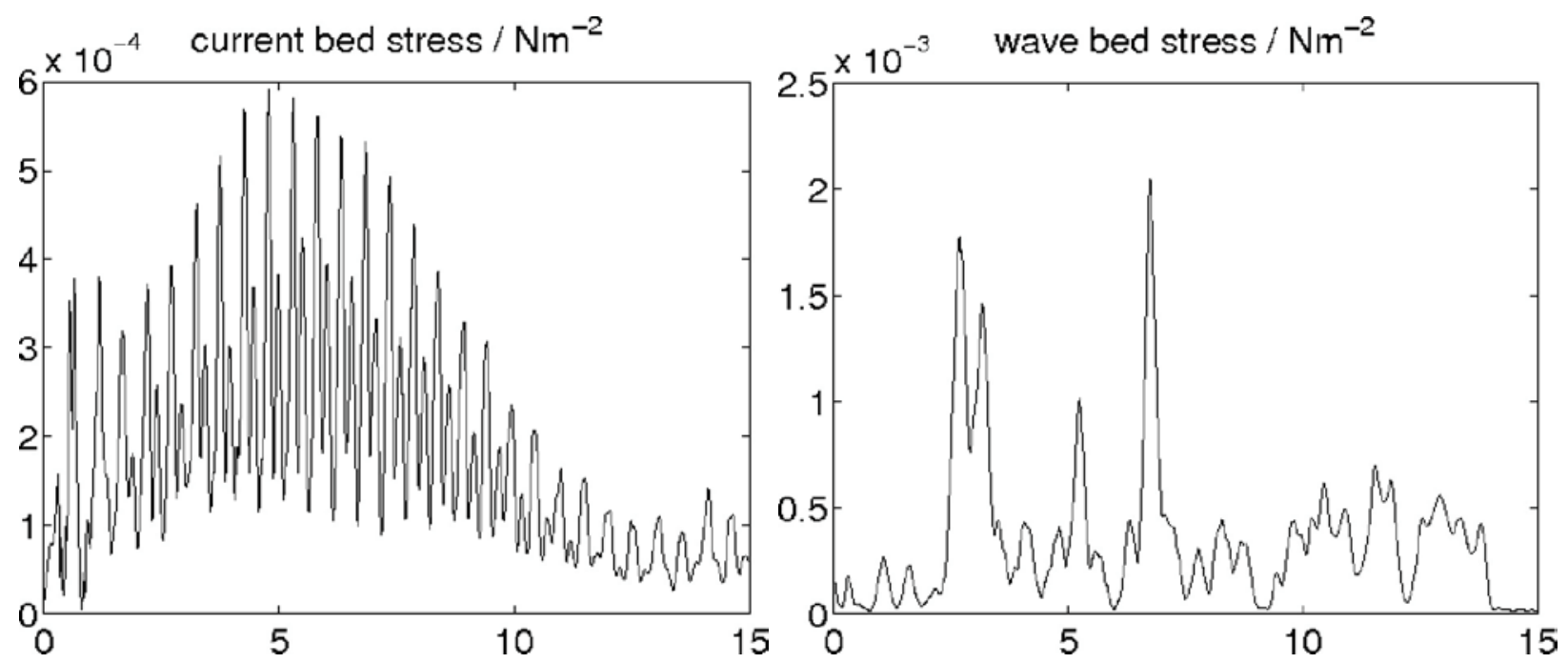

Figure 7.1 Time series of bottom stress produced by currents (left) and waves (right). 
The POLCOMS-WAM-SPM model is implemented in the Irish Sea, forced by waves, tide and surge at the open boundaries, and hourly meteorological fields at the surface. We use two different grain sizes, defined by their settling velocities, $W=2.0 \times 10^{-2} \mathrm{~m} / \mathrm{s}$ represents a medium sand, and $W=1.0 \times 10^{-3} \mathrm{~m} / \mathrm{s}$ a fine mud. This range spans the range of bed composition in the Irish Sea.

\subsection{Results}

While the maximum bed stresses associated with tidal currents alone are periodic and predictable, those from waves are more sporadic - relating to the prevailing meteorological conditions. The enhanced wave bed stress, observed during storm periods, is large enough to 'swamp' the background tidal stresses. These large 'benthic storms' have an important impact on SPM, and are able to resuspend much larger quantities of sediment in a single event than is moved by the gentler tidal motions. Figure 7.1 shows how bottom stress is enhanced during the course of a storm in the North Sea and Figure 7.2 shows the effect of periodic tidal stresses on the supply of bottom sediment to the water column.

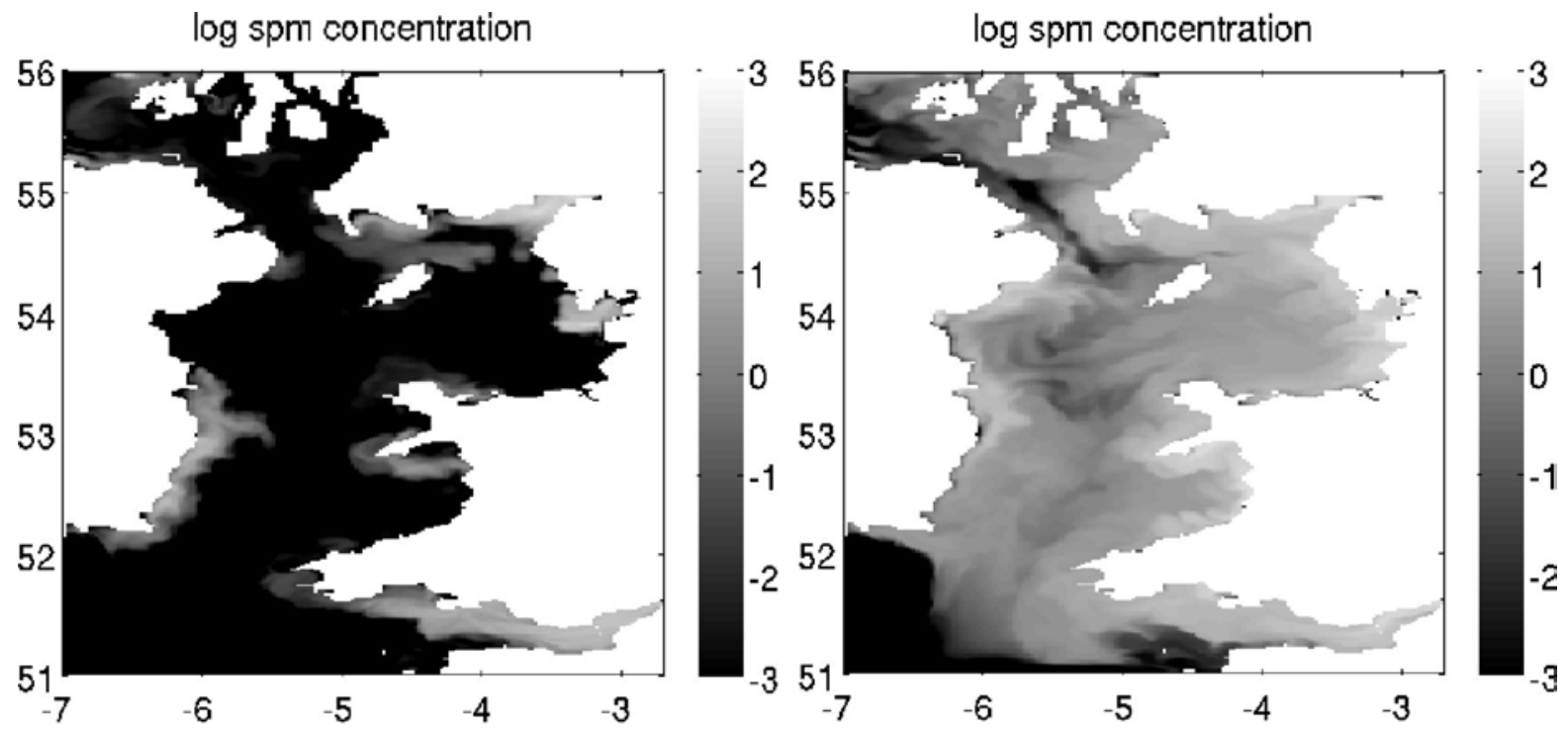

Figure 7.2 Maps of suspended particulate matter after 1 week of simulation in an unenhanced, current only simulation (left) and wave enhanced (right).

\subsection{Discussion and conclusions}

There is a continuing trend to encourage interdisciplinary modelling, particularly in shelf seas, and at the land-ocean boundary, where complex physical processes control biological productivity and sediment transport. Therefore it is important that separate model components are properly coupled, so that their interactions better reflect the real-world behaviour.

Further work in this area will follow that of Sykes and Barciela (In preparation), who include aggregationdisaggregation into their sediment model, finding an improved distribution of suspended particulate matter, when compared with ocean_colour satellite images. 


\subsection{References}

Holt, J.T. \& James, I.D. (1999). A simulation of the southern North Sea in comparison with measurements from the North Sea Project Part 2 Suspended Particulate Matter. Continental Shelf Research. 19, No. 12, 1617-1642

Holt, J.T. \& James, I.D. (2001). An s-coordinate density evolving model of the North West European Continental Shelf. Part 1. Model description and density structure. Journal of Geophysical Research - Ocean, 106, No. C7, 14015-14034.

Sykes, P.A. \& Barciela, R.M. (In preparation). Assessment and Development of a Sediment Model within an Operational System.

Soulsby, R. (1997). Dynamics of marine sands: a manual for practical applications. Thomas Telford Publications, London, 249pp. 


\section{The migration of large scale bed forms in an estuarine environment - Oliver Way, Alan Davies and Paul Bell}

\subsection{Introduction}

The Dee Estuary (Figure 8.1) is macrotidal, with a peak spring tidal range in excess of $10 \mathrm{~m}$. The large tidal range and associated strong currents make the Dee Estuary a very dynamic system with large fluxes of sediment. River canalisation and land reclamation between 1700 s and early 1900 s significantly altered the hydrodynamic and sedimentological conditions. This caused the main navigation channel to migrate from the eastern side to the western shore of the estuary. Continuous monitoring of large scale bedforms in the estuary mouth and the wave and current forces responsible for the migration of these features will help to understand the evolution of the estuary. Remote measurements of dune height will allow more accurate model simulations of bedform and current interaction.

\subsection{Method}

The TELEMAC modelling system is used to show the driving forces behind bedform migration, including the effect of waves and tidal currents.

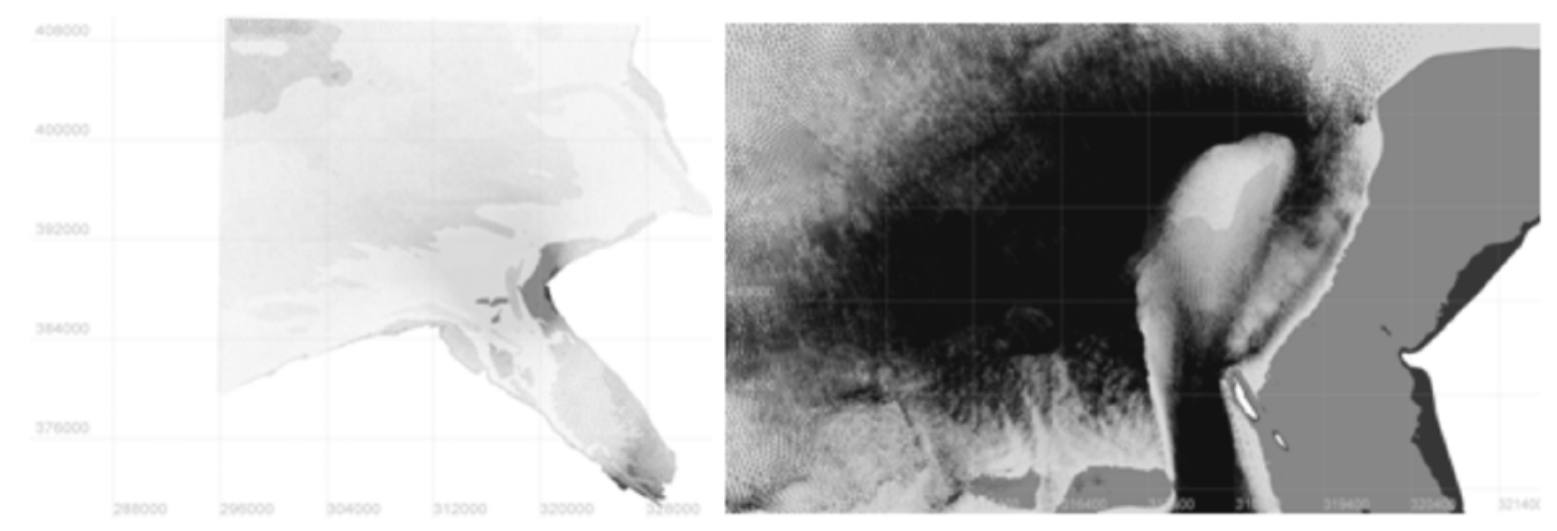

Figure 8.1 TELEMAC model domain extends out into Liverpool Bay. Waves and currents are simulated over this grid to show sediment transport in the mouth of the estuary.

Images from the X-Band marine radar station deployed on Hilbre Island (Figure 8.1) are used to infer bathymetry and to track the movement of wave breaker patterns over large intertidal sand dunes. The movement of sand dunes should correspond to existing bedload transport formulae (Van den Berg, 1987). A 2-D cross correlation tracking algorithm (Bell, 2005) is applied to monthly averaged radar images to estimate dune migration from wave breaker patterns. This method will help to establish whether or not large scale bedforms are actually moving in selected areas of the Dee where migration is expected. A variation on the 'waterline' method (Figure 8.2, Mason et al., 1999) is also used to show intertidal bathymetry from the Xband radar images. 


\subsection{Results}
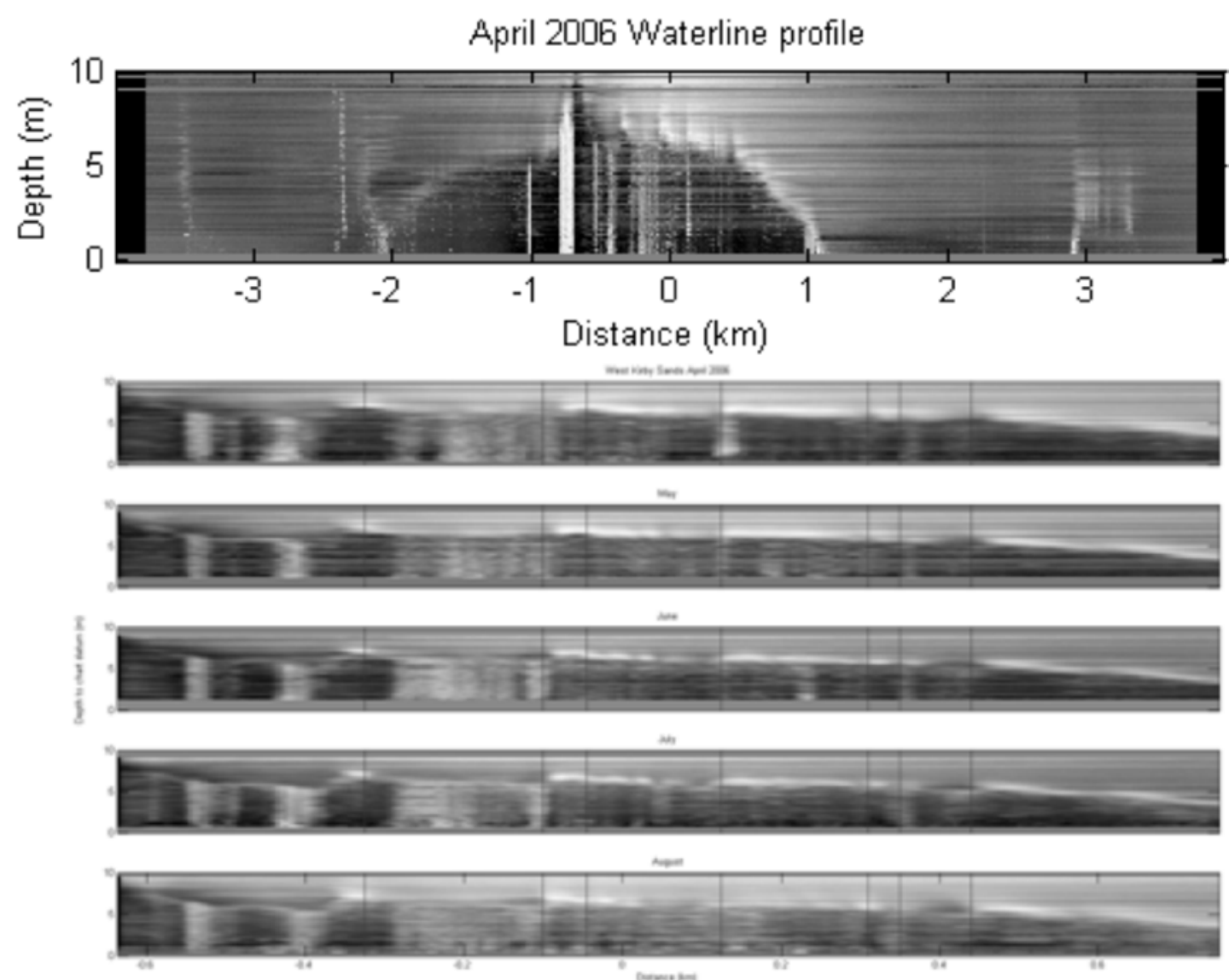

October to November 2006 migration $=3.6 \mathrm{~m}$

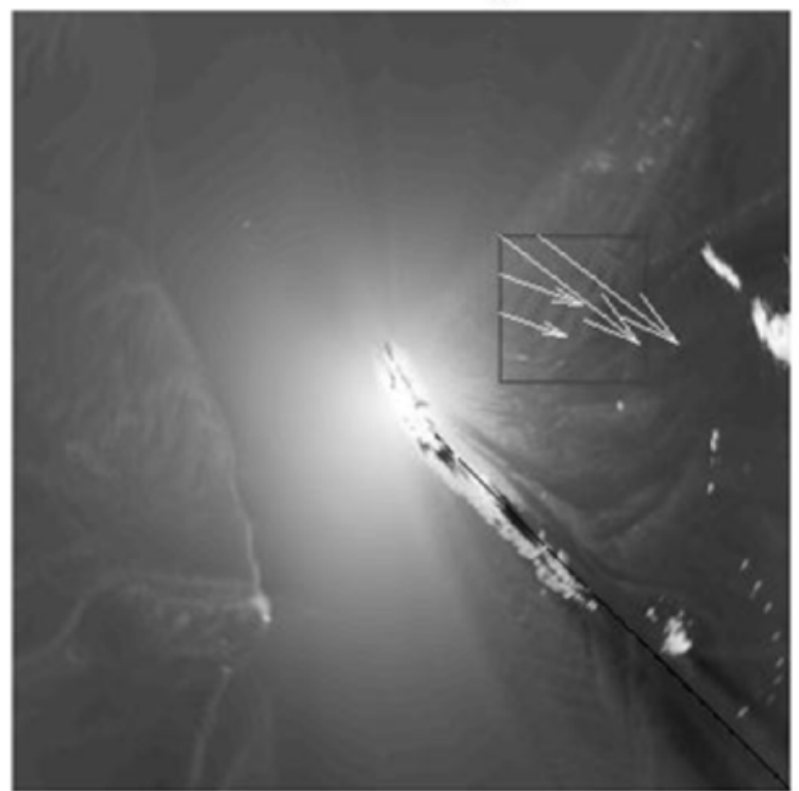

Figure 8.2 Waterline profiles show large bedforms migrating into West Kirby Sands. Motion tracking with a cross correlation algorithm approximates the migration rate into the estuary. 
This research will ultimately aim to show if remotely measuring the dimensions of sand dunes will enable an accurate calculation of bedform roughness to be made and included into modelling systems such as TELEMAC. The physical bedform roughness of sand dunes is roughly on the order of half the bedform height (van Rijn, 1993), therefore an appropriate method of radar image analysis should be determined to provide the most accurate bedform profile.

\subsection{References}

Bell, P.S. (2005). Remote determination of bathymetric changes using ground based radar. University of Wales Bangor, School of Ocean Sciences, UK, Ph.D. Thesis. 213pp.

Mason, D.C., Amin, M., Davenport, I.J., Flather, R.A., Robinson, G.J. \& Smith, J.A. (1999). Measurement of recent intertidal sediment transport in Morecambe Bay using the waterline method. Estuarine, Coastal and Shelf Science, 49, No. 3, 427-456.

Van den Berg, J.H. (1987). Bedform migration and bed load transport in some rivers and tidal environments. Sedimentology, 34, No. 4, 681-698.

Van Rijn, L.C. (1993). Principles of sediment transport in rivers, estuaries and coastal areas. Aqua Publications, Amsterdam, The Netherlands, 633pp. 


\section{Acknowledgements}

Without the support from the conference steering committee (Alistair Borthwick, Nick Dodd, Suzana llic, Robert Nicholls, Richard Simons) and local organising committee (Laurent Amoudry, Paul Bell, Alejandro Souza) the YCSEC'11 would not have been such a success. Tim Chesher and Andrew Willmott are thanked for giving up time to speak to early-career participants about their work. The generosity of sponsors (babcock, BIGF, Cambridge University Press, Cefas, defra, fugro, ice, IMAREST, International Ocean Systems, NOC, Nortek UK, North West \& North Wales Coastline, Ocean Business, Planet Ocean Ltd, RS AQUA Ltd, Sequoia, TSI, YSI) was greatly appreciated to keep the conference affordable for the majority of students and enabling a conference dinner and field trip to be included. 